\title{
Independent component analysis of interictal fMRI in focal epilepsy: comparison with general linear model- based EEG-correlated fMRI
}

Citation for published version (APA):

Rodionov, R., de Martino, F., Laufs, H., Carmichael, D. W., Formisano, E., Walker, M., Duncan, J. S., \& Lemieux, L. (2007). Independent component analysis of interictal fMRI in focal epilepsy: comparison with general linear model-based EEG-correlated fMRI. Neuroimage, 38(3), 488-500.

https://doi.org/10.1016/j.neuroimage.2007.08.003

Document status and date:

Published: 01/01/2007

DOI:

10.1016/j.neuroimage.2007.08.003

Document Version:

Publisher's PDF, also known as Version of record

Document license:

Taverne

Please check the document version of this publication:

- A submitted manuscript is the version of the article upon submission and before peer-review. There can be important differences between the submitted version and the official published version of record.

People interested in the research are advised to contact the author for the final version of the publication, or visit the DOI to the publisher's website.

- The final author version and the galley proof are versions of the publication after peer review.

- The final published version features the final layout of the paper including the volume, issue and page numbers.

Link to publication

\footnotetext{
General rights rights.

- You may freely distribute the URL identifying the publication in the public portal. please follow below link for the End User Agreement:

www.umlib.nl/taverne-license

Take down policy

If you believe that this document breaches copyright please contact us at:

repository@maastrichtuniversity.nl

providing details and we will investigate your claim.
}

Copyright and moral rights for the publications made accessible in the public portal are retained by the authors and/or other copyright owners and it is a condition of accessing publications that users recognise and abide by the legal requirements associated with these

- Users may download and print one copy of any publication from the public portal for the purpose of private study or research.

- You may not further distribute the material or use it for any profit-making activity or commercial gain

If the publication is distributed under the terms of Article $25 \mathrm{fa}$ of the Dutch Copyright Act, indicated by the "Taverne" license above, 


\title{
Independent component analysis of interictal fMRI in focal epilepsy: Comparison with general linear model-based EEG-correlated fMRI
}

\author{
R. Rodionov ${ }^{\mathrm{a}, \mathrm{b}, *}$ F. De Martino, ${ }^{\mathrm{c}}$ H. Laufs, ${ }^{\mathrm{a}, \mathrm{b}, \mathrm{d}}$ D.W. Carmichael, ${ }^{\mathrm{a}, \mathrm{b}}$ E. Formisano, ${ }^{\mathrm{c}}$ \\ M. Walker, ${ }^{a, b}$ John S. Duncan, ${ }^{a, b}$ and L. Lemieux ${ }^{a, b}$ \\ ${ }^{\mathrm{a} D e p a r t m e n t}$ of Clinical and Experimental Epilepsy, Institute of Neurology, University College of London Queen Square, London WC1N 3BG, UK \\ ${ }^{\mathrm{b}}$ MRI Unit, National Society for Epilepsy, Chesham Lane, Chalfont St. Peter, Buckinghamshire SL9 ORJ, UK \\ ${ }^{\mathrm{c} D e p a r t m e n t ~ o f ~ C o g n i t i v e ~ N e u r o s c i e n c e s, ~ F a c u l t y ~ o f ~ P s y c h o l o g y, ~ U n i v e r s i t y ~ o f ~ M a a s t r i c h t, ~ M a a s t r i c h t, ~ T h e ~ N e t h e r l a n d s ~}$ \\ ${ }^{\mathrm{d}}$ Brain Imaging Center and Klinik für Neurologie, Johann Wolfgang Goethe-Universität, Theodor-Stern-Kai 7, 60590 Frankfurt am Main, Germany
}

Received 2 May 2007; revised 27 June 2007; accepted 6 August 2007

Available online 17 August 2007

\begin{abstract}
The general linear model (GLM) has been used to analyze simultaneous EEG-fMRI to reveal BOLD changes linked to interictal epileptic discharges (IED) identified on scalp EEG. This approach is ineffective when IED are not evident in the EEG. Data-driven fMRI analysis techniques that do not require an EEG derived model may offer a solution in these circumstances. We compared the findings of independent components analysis (ICA) and EEG-based GLM analyses of fMRI data from eight patients with focal epilepsy. Spatial ICA was used to extract independent components (IC) which were automatically classified as either BOLD-related, motion artefacts, EPI-susceptibility artefacts, large blood vessels, noise at high spatial or temporal frequency. The classifier reduced the number of candidate IC by $78 \%$, with an average of 16 BOLD-related IC. Concordance between the ICA and GLM-derived results was assessed based on spatio-temporal criteria. In each patient, one of the IC satisfied the criteria to correspond to IED-based GLM result. The remaining IC were consistent with BOLD patterns of spontaneous brain activity and may include epileptic activity that was not evident on the scalp EEG. In conclusion, ICA of fMRI is capable of revealing areas of epileptic activity in patients with focal epilepsy and may be useful for the analysis of EEG-fMRI data in which abnormalities are not apparent on scalp EEG. (C) 2007 Elsevier Inc. All rights reserved.
\end{abstract}

\section{Introduction}

In some patients with drug resistant focal epilepsy, surgical resection offers the possibility of seizure control. If the focus is not adequately localized non-invasively, with MRI and scalp EEG, intra-cranial electroencephalography (icEEG) using subdural or

\footnotetext{
* Corresponding author. Department of Clinical and Experimental Epilepsy, Institute of Neurology, University College of London Queen Square, London WC1N 3BG, UK.

E-mail address: r.rodionov@ion.ucl.ac.uk (R. Rodionov).

Available online on ScienceDirect (www.sciencedirect.com).
}

intracerebral electrodes may be necessary to define the zone of seizure onset. This procedure is invasive and may fail to identify the epileptogenic zone, in part because of the limited spatial sampling of intracranial electrodes.

Functional MRI enables the non-invasive observation of brain activity with relatively high spatial resolution over the whole brain. EEG-correlated fMRI (EEG-fMRI) has shown promise in epilepsy. Using EEG-fMRI, regions of brain activation and deactivation have been demonstrated in relation to interictal and ictal epileptic discharges, providing a new form of localizing information (Hamandi et al., 2004; Gotman et al., 2006; Laufs and Duncan, 2007). It has been suggested that this type of information could be useful to plan or even to remove the need for intracranial EEG in some cases (Allen et al., 2000; Lemieux et al., 2001). The standard analysis of EEG-fMRI data is based on the identification of interictal epileptiform discharges (IED) on scalp EEG which are used to build a general linear model (GLM) of the fMRI signal changes. In brief, a model is obtained by convolution of the EEG events, which are represented as stick functions or blocks, with a hemodynamic response function; maps showing regions of significant IED-related change are obtained through voxel-wise fitting of the model and application of appropriate statistical thresholds (Lemieux et al., 2001; Benar et al., 2002; Salek-Haddadi et al., 2003; Hamandi et al., 2004; Gotman et al., 2006).

Reliance on scalp EEG for the modeling of BOLD changes throughout the brain is useful to demonstrate hemodynamic changes related to specific EEG patterns but has limitations, because:

(1) The number of EEG events recorded during a 10-40 minute fMRI acquisition must be sufficient for efficient model estimation. The EEG/GLM approach cannot be used when no or too few interictal epileptic discharges (IED) are detected during the fMRI experiment, which is not uncommon (Krakow et al., 1999; Gotman et al., 2004; Salek-Haddadi et al., 2006; Di Bonaventura et al., 2006). 
(2) The scalp EEG has limited sensitivity, particularly for activity originating deep in the brain (Tao et al., 2005; Ray et al., 2007), with a resultant bias towards the detection of superficial IED. Furthermore it is well known that epileptic activity originating from deep brain structures in many cases cannot be recorded on the scalp EEG (Niedermeyer and Lopes da Silva, 2004).

(3) Any epilepsy-related activity which is not detected by scalp EEG will not be modeled, with a potentially significant impact on the technique's sensitivity.

In such circumstances, data-driven fMRI data analysis techniques may provide a way forward (Morgan et al., 2004) as they are not constrained by a fixed hypothesis. This may be particularly beneficial in cases where no hypothesis is available for example in the absence of discharges on the scalp EEG.

ICA is increasingly recognized as a useful fMRI data-driven analysis tool (McKeown et al., 1998; Formisano et al., 2004; Beckmann et al., 2005). Not being reliant on prior hypotheses, ICA of fMRI has the potential to identify a greater proportion of the BOLD signal variations. The main advantage of this method is that it represents the original functional time series as a set of independent components (IC), which may separate meaningful neurophysiological sources and artefacts. However, the lack of a prior hypothesis and the potentially large number of IC generated render interpretation of the results difficult (Formisano et al., 2002; Beckmann and Smith, 2004). Some authors combine ICA with a GLM for fMRI analysis, using IC as GLM regressors (McKeown, 2000; Hu et al., 2005; Mirsattari et al., 2006). Since correlation with an experimental paradigm is a criterion for selection of IC to build a GLM, the core problem of IC interpretation is not tackled in these techniques. The idea of sequential application of spatial and temporal ICA in order to reveal epilepsy-related IC has been suggested, but not evaluated (Chen et al., 2006).

An automated characterization technique has been introduced and implemented to reduce the number of meaningful IC that require interpretation (De Martino et al., 2007). In this method, the classification of patterns as BOLD-like relies on a set of spatial and temporal characteristics derived from data acquired in normal healthy subjects. In the context of epilepsy, it has been suggested that the time course of IED-related BOLD changes may deviate from the canonical shape (Diehl et al., 2003; Salek-Haddadi et al., 2003). However, the IED-related response has been shown to be primarily canonical (Salek-Haddadi et al., 2006; Lemieux et al., 2007) and any deviation is likely to reflect scalp EEG bias rather than coupling or vascular abnormalities (Lemieux et al., 2007; Hawco et al., 2007).

Our goal is to assess the potential of ICA to identify epileptic activity in fMRI datasets acquired in patients with focal epilepsy by comparing the results with the patterns identified using a GLMbased approach (based on EEG abnormalities seen on scalp EEG) in cases with clear electro-clinical localization. We applied the method of spatial ICA to fMRI data and used an automated classification approach (De Martino et al., 2007), independent of the EEG, to reduce the number of components that are likely to represent epileptic activity. We have chosen to use the classifier as currently trained using data from healthy subjects for this initial study, based on the general normality of the spike-related BOLD response as described above. Then we determined whether the selected components included IED-related spatio-temporal patterns by comparing them with the results of the EEG-based GLM analysis of the same data, resulting in a set of IED-related independent components.

\section{Data}

Sixty-three patients with focal epilepsy underwent EEGcorrelated fMRI (Salek-Haddadi et al., 2006). Eight patients (Table 1) were selected based on the following criteria: (1) clear-cut localization and lateralization on the basis of concordance between clinical seizures, EEG and structural MRI; (2) the localization of the GLM-derived fMRI signal change was concordant with other electro-clinical data (Table 1) (Salek-Haddadi et al., 2006). All patients gave written informed consent (Joint Ethics Committee of the National Hospital for Neurology and Neurosurgery and Institute of Neurology).

BOLD sensitive EPI images (TE/TR 40/3000, $21 \times 5$ contiguous, interleaved slices, FOV $24 \times 24 \mathrm{~cm}, 64 \times 64$ matrix) were acquired, with a $1.5 \mathrm{~T}$ Horizon Echospeed (General Electric, Milwaukee, USA) MRI scanner with continuous, simultaneous EEG and ECG recording. In seven subjects, 700 scans were acquired. In one case 450 scans were acquired due to a seizure. High-resolution T1 weighted MR images were also acquired (Fast

Table 1

Patient data and EEG-fMRI GLM results

\begin{tabular}{|c|c|c|c|c|c|}
\hline Case \# & $\begin{array}{l}\text { Case \# in Salek-Haddadi } \\
\text { et al., } 2006\end{array}$ & $\begin{array}{l}\text { Case \# in Hamandi } \\
\text { et al., } 2005\end{array}$ & Aetiology & $\begin{array}{l}\text { EEG-fMRI GLM } \\
\text { activation localization }\end{array}$ & Surgery outcome \\
\hline 1 & 13 & $\mathrm{~B}$ & TLE, L-HS & LH, cuneus & 4 \\
\hline 2 & 2 & $\mathrm{D}$ & $\mathrm{MCD}$ & $\begin{array}{l}\text { Diffused R mid-temporal } \\
\text { and frontal }\end{array}$ & $\mathrm{n} / \mathrm{a}$ \\
\hline 3 & 35 & $\mathrm{H}$ & MCD & L temporal & $\mathrm{n} / \mathrm{a}$ \\
\hline 4 & 12 & $\mathrm{~F}$ & TLE, L-HS & $\begin{array}{l}\mathrm{L} \text { anterior } \\
\text { temporal }\end{array}$ & 2 \\
\hline 5 & 31 & $\mathrm{C}$ & $\mathrm{MCD}$ & $\begin{array}{l}\mathrm{R} \text { parietal and } \\
\text { temporo-parietal }\end{array}$ & $\mathrm{n} / \mathrm{a}$ \\
\hline 6 & 6 & $\mathrm{~A}$ & Chronic encephalitis & L temporal parietal & 9 \\
\hline 7 & 23 & $\mathrm{G}$ & Post-traumatic & $\mathrm{R}$ frontal and $\mathrm{R}$ parietal & $\mathrm{n} / \mathrm{a}$ \\
\hline 8 & 3 & $\mathrm{E}$ & MCD & L temporal & $\mathrm{n} / \mathrm{a}$ \\
\hline
\end{tabular}

The EEG-fMRI GLM activation localization was taken from Salek-Haddadi et al. (2006). Surgery outcome: number of post-surgical years of seizure freedom. Abbreviations: TLE_temporal lobe epilepsy; L-HS—left hippocampus sclerosis; MCD_malformation of cortical development; LH_left hippocampus; $\mathrm{R} / \mathrm{L}$ —right/left; n/a_no information available. 
Inversion Recovery [prepared] Spoiled Gradient Recalled [IRSPGR]: TI/TR/TE, 450/15/4.2 (ms), flip angle $25^{\circ} ; 1241.5 \mathrm{~mm}-$ thick coronal slices; matrix, $256 \times 192$ voxels, $24 \times 18 \mathrm{~cm}$ field of view; scan time, $7 \mathrm{~min}$ ). Patients were asked to rest with their eyes shut and keep their head still.

The EEG was recorded using in-house equipment. Ten channels of common reference EEG in a bi-temporal chain Fp2/Fp1, F8/F7, T4/T3, T6/T5, O2/O1, Fz (ground) and $\mathrm{Pz}$ as the reference, according to the international 10:20 system and two channels of ECG were recorded inside the MRI scanner with online artefact removal (Allen et al., 1998; Allen et al., 2000; Lemieux et al., 2001). The EEG was reviewed off-line and the onset of IED identified by two trained observers who reached consensus on the set of marked IED.

\section{Methods}

The fMRI data were analyzed using two methods and the results compared: (1) ICA without reference to the simultaneously recorded EEG, and (2) the EEG-based GLM approach (IEDcorrelated fMRI).

\section{ICA of fMRI data}

Spatial independent component analysis was performed with Brain Voyager QX software (Brain Innovation, Maastricht,
Netherlands). The mathematical details of the ICA for fMRI are described elsewhere (McKeown et al., 1998, 2003). Details of implementation of ICA in BrainVoyager QX are described in Formisano et al. (2004).

In summary, the ICA decomposition can be expressed as:

$X=A S$

where $X$ is the measured fMRI signal, $S$ the spatial maps of the decomposition and $A$, the time courses defining the relative weighting of the spatial maps throughout the experiment. We estimated $A$ and $S$, using the hierarchical (deflation) mode of the FastICA algorithm (Hyvärinen, 1999), after reduction of the temporal dimension of the data set with principal component analysis to 80 dimensions. This number of dimensions was selected after a preliminary analysis in which we performed consecutive decompositions and verified that the number of IC classified as BOLD was stable and spatiotemporal characteristics of those IC did not change significantly while increasing the number of IC in the decomposition.

\section{Classification of independent components}

After decomposition automatic IC classification was applied (De Martino et al., 2007) resulting in the following set of labels: (1) the 'BOLD' class, which included components that are thought to consistently reflect task-related, transiently task-related and brain
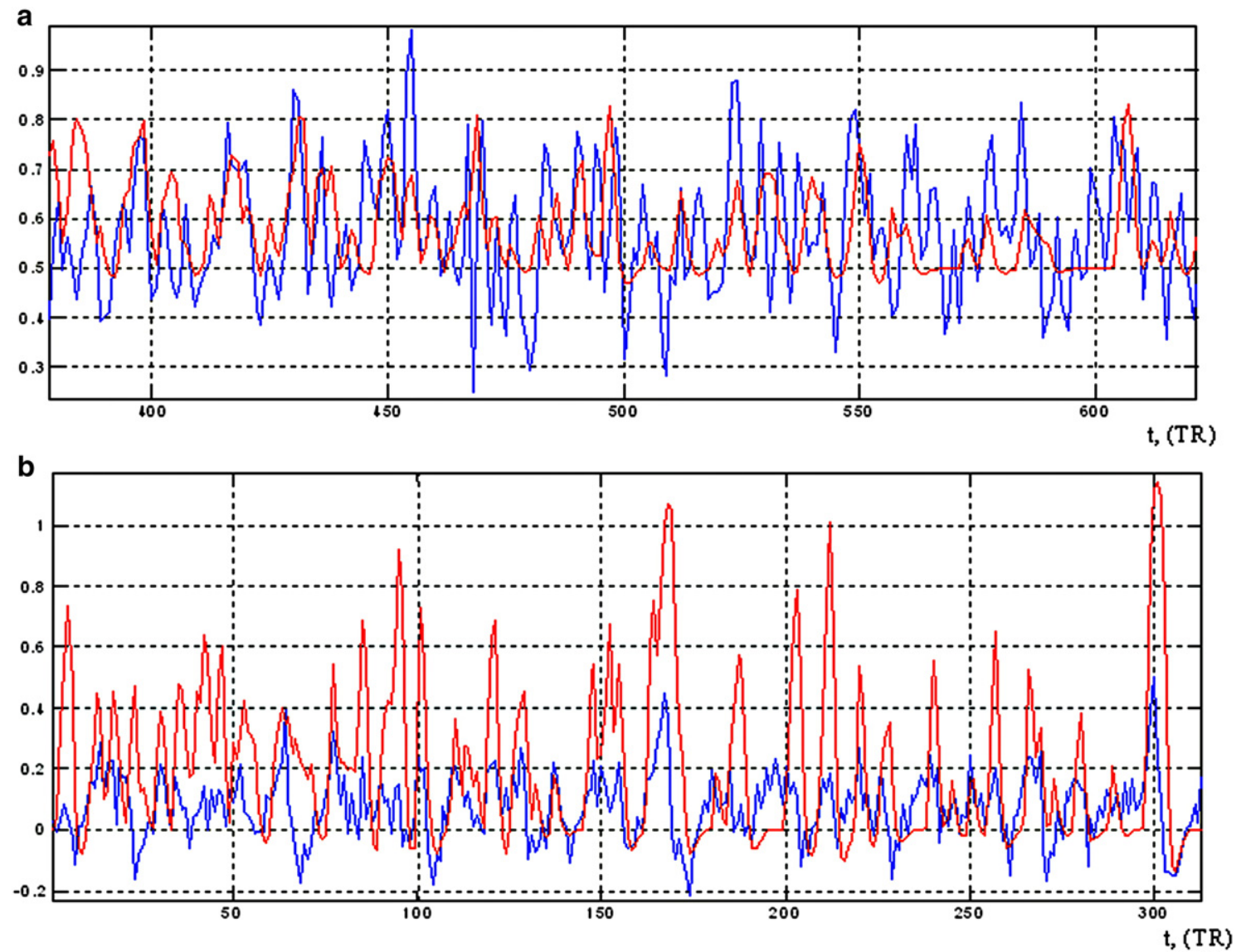

Fig. 1. Fragment of the time course of IC (blue) significantly correlated with corresponding IED-related (GLM) regressor (red) for case $\# 4$ (a) $(R=0.26$, $p=6.6 \mathrm{e}-12)$ and case \#7 (b) $(R=0.3, p=2.6 \mathrm{e}-15)$. (For interpretation of the references to colour in this figure legend, the reader is referred to the web version of this article.) 
state-related (e.g. default state) neuronal activity; (2) residual motion artefacts; (3) EPI-susceptibility artefacts; (4) physiological noise; (5) noise at high spatial frequency; and (6) noise at temporal high frequency.

The classifier is the result of training with a dataset from healthy volunteers (De Martino et al., 2007) and is designed to be inclusive rather than restrictive with respect to BOLD components in order to reduce the probability of misclassification of BOLDrelated IC. Furthermore, it has the ability to reveal stereotypical components of normal brain activity such as the so-called 'default mode' network (Schmithorst and Brown, 2004; Greicius and Menon, 2004) and sensory components reflecting connectivity during rest (Van de Ven et al., 2004). The BOLD components were further classified into the following sub-types by visual inspection:

1. Stereotypical of normal brain activity;

2. Misclassified;

3. Other.
The misclassified type contains those with patterns corresponding to one of the following effects (De Martino et al., 2007): motion, blood vessels, spatially distributed and high frequency noise. We hypothesized that IC classified as 'Other' would contain IC related to IED and form the set of candidate components.

\section{GLM-based analysis of EEG-fMRI data}

A GLM-based analysis (Frackowiak et al., 2003) of the fMRI datasets was performed with Brain Voyager QX software based on the identification of IED on the scalp EEG. Two trained observers coded the EEGs together resulting on a consensus for the entire recordings. Data pre-processing consisted of scan realignment and smoothing using an $8 \mathrm{~mm}$ kernel (Frackowiak et al., 2003). The GLM comprised HRF-convolved IED-related effects (SalekHaddadi et al., 2006), motion effects (a combination of Volterra expansion of the 6 scan realignment parameters) (Friston et al., 1996; Lemieux et al., 2007) and scan nulling of head jerks (Salek-
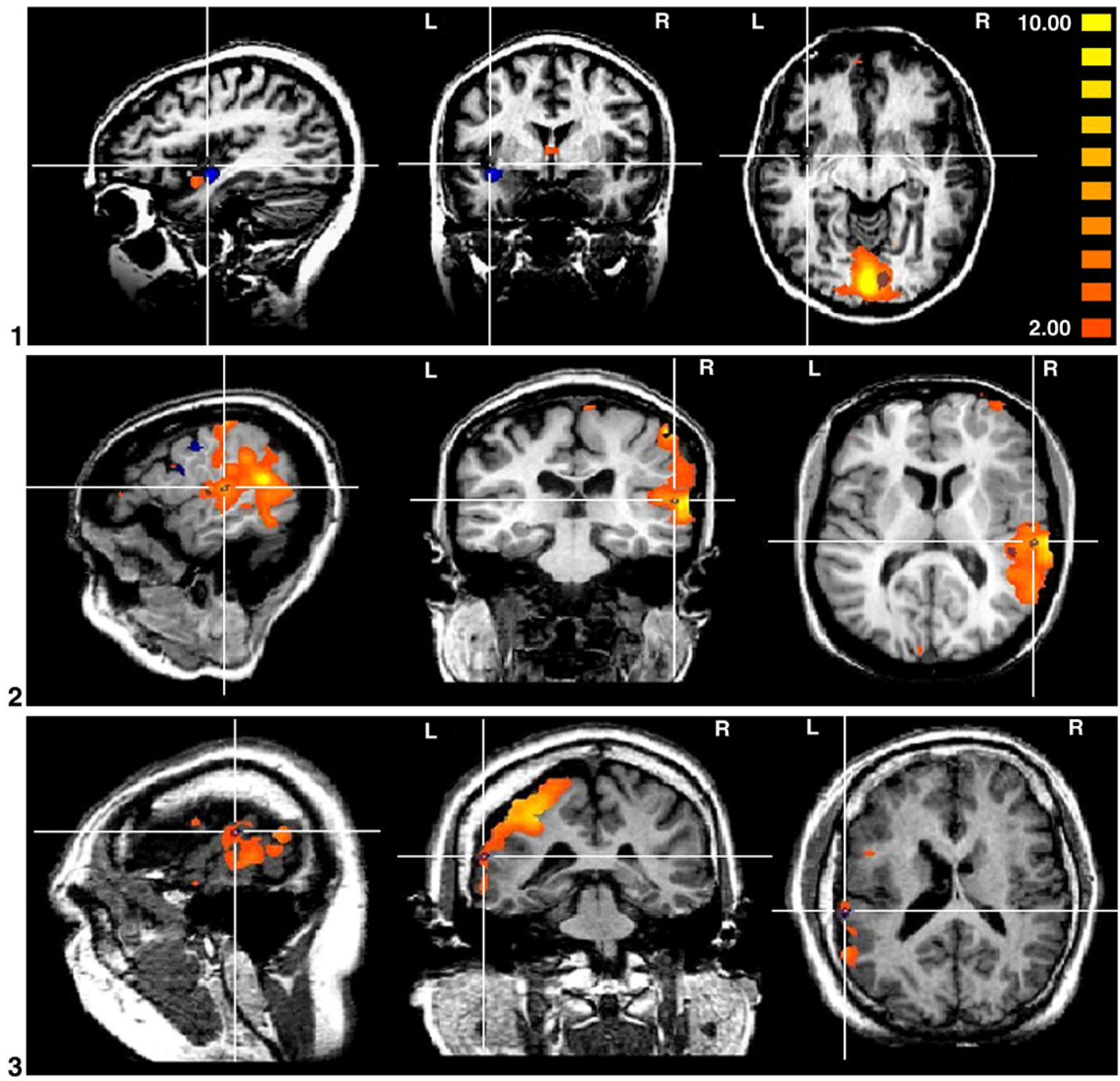

Fig. 2. Illustration of SPM (dark blue) and matching IC maps (orange) for all cases, overlap is shown in purple shades. Cursor is located so as to define sections that best illustrate overlap. The thresholds for cases $1-7$ are $z>2.0$ for the IC maps and $p<0.05$ (corrected) for the SPM. The thresholds for the case 8 are chosen as $z>1.6$ for the IC map and $p<0.05$ (corrected) for SPM to facilitate visualization (only two voxels survive $z>2.0$ threshold). In case \#4 the map of the misclassified IC is shown (see explanation in the Discussion section). The numbers for each set of projections correspond to \# of case in Tables 1 and 2. (For interpretation of the references to colour in this figure legend, the reader is referred to the web version of this article.) 

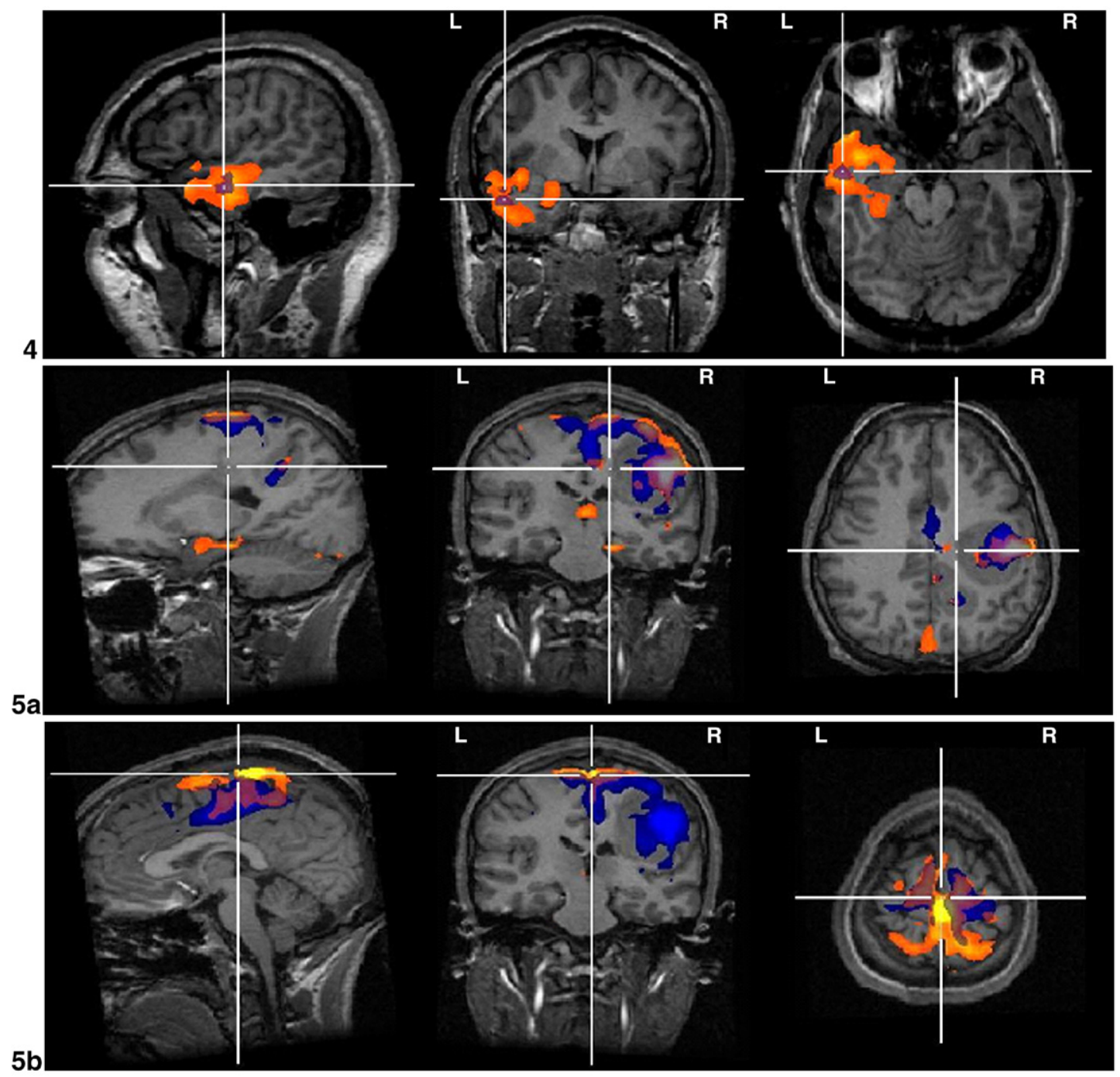

Fig. 2 (continued).

Haddadi et al., 2006; Lemieux et al., 2007). Statistical parametric maps (SPM) of activation were obtained by testing for positive BOLD changes using $p<0.05$ (Bonferroni corrected). The set of EPI images was co-registered with the $\mathrm{T} 1$ volume dataset scaled to Talairach space by means of rigid body transformation for visualization. In these cases, positive BOLD responses were found consistently corresponding to the electro-clinical findings in line with our findings in a larger series (Salek-Haddadi et al., 2006). This is in contrast to the negative BOLD changes, which are generally more remote from the presumed generator. Therefore, only positive IED-related BOLD changes were considered in this study.

\section{Comparison of ICA and GLM-based results}

The values of the particular IC map represent the relative amount a given voxel is modulated by the activation of that component. To identify significantly contributing voxels, ICA maps were scaled to the spatial $z$-scores, computed as the number of standard deviations from the map mean (McKeown et al., 1998). A threshold of $z>2.0$ was used for visualization of the IC maps and volumetric comparison of the IC maps and SPM.
Each BOLD component was compared with the GLM solution (positive BOLD changes only). Components that matched the following criteria were classified as matching (IED-related): (1) temporal correlation between component time course and IEDderived regressor with $p<0.01$ (Figs. 1 and 2) spatial overlap of the thresholded IC map $(z>2.0)$ exceeding $10 \%$ of spatial extent of GLM-derived clusters (Fig. 2). The value of $10 \%$ of spatial overlap was chosen based on preliminary tests on 3 datasets.

\section{Results}

The ICA decompositions are summarized in Table 2. The number of BOLD components ranged between 11 and 24 (mean: 16, median: 15 ) representing an average of $22 \%$ of the total (Table 2). In six cases one, and in one case, two of the candidate components matched the GLM-derived result according to the set criteria. In one case, \#4, no BOLD component was found to meet the matching criteria (Table 2, Fig. 2).

In accordance with previous studies of the resting state in healthy subjects, components characteristic of the so-called resting state networks (RSN) were found consistently in the group studied. 

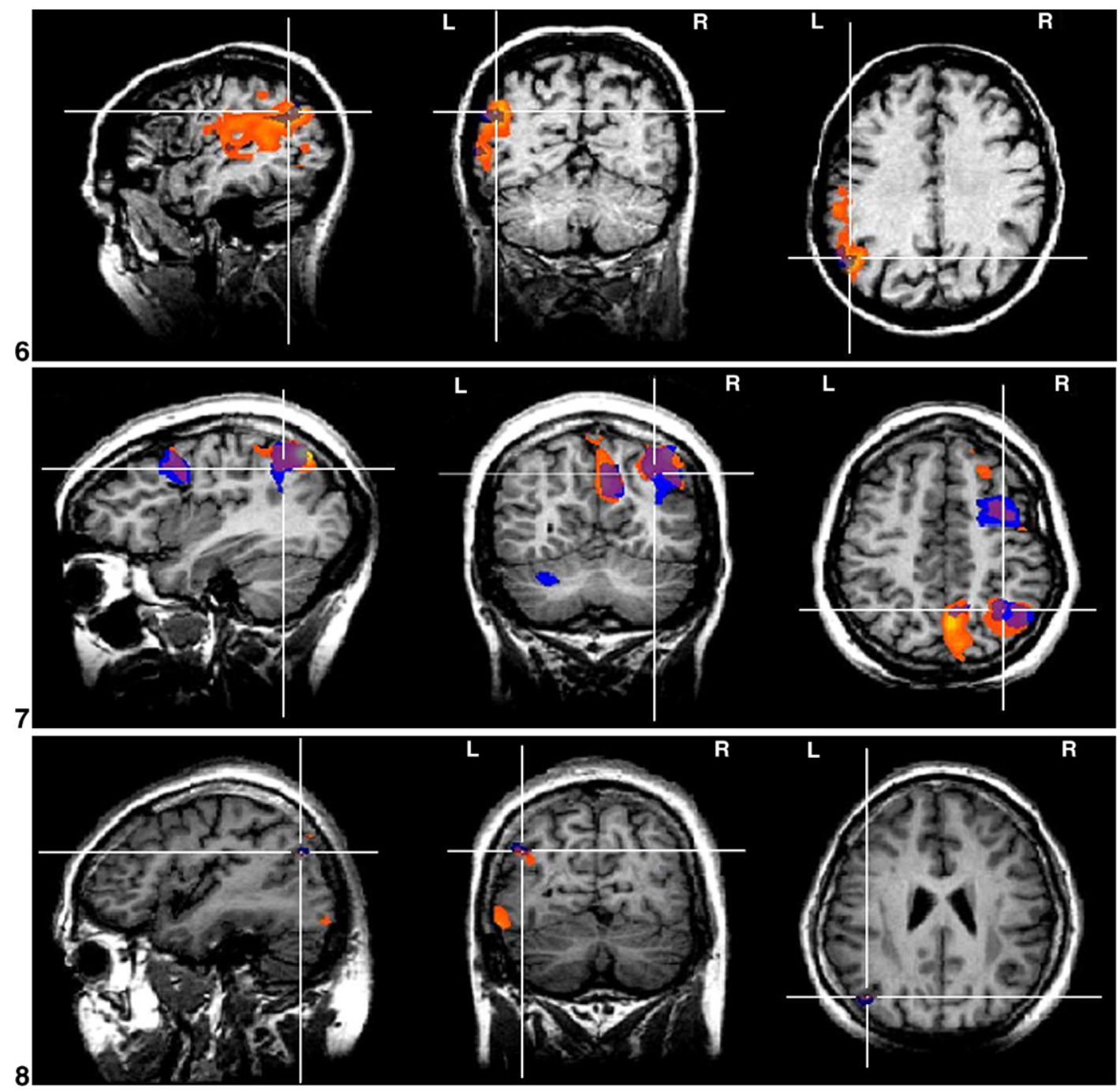

Fig. 2 (continued).

These involved sensory-motor, visual and auditory areas and the so-called default mode network (DMN: precuneus and frontooccipital areas), the latter illustrated in Fig. 3.

\section{Case reports}

In accordance with the selection criteria used for this study, the result of the GLM analysis was concordant with the presumed or confirmed focus for all cases (Salek-Haddadi et al., 2006).

\section{Case 1}

Patient with left hippocampal sclerosis and left temporal IEDs. The GLM analysis revealed a left temporal activation with an additional smaller activation cluster in the occipital region. The single matching IC consisted of a cluster in the left hippocampus and a larger one in the occipital region, either overlapping or adjacent to the GLM pattern (Fig. 2.1).

\section{Case 2}

Patient with an extensive malformation of cortical development in the right hemisphere, involving predominantly the parietal lobe but extending to the occipital and frontal lobes, plus focal atrophy of the left parietal lobe. GLM analysis revealed an area of right frontal and mid-temporal activation linked to right temporal IEDs. The GLM analysis for this patient has a lower level of significance possibly due to the large degree of motion (Salek-Haddadi et al., 2006). One IC matched the GLM result, covering the parietal part of the lesion and corresponding clusters of GLM activation (Fig. 2.2).

\section{Case 3}

Patient with left parietal polymicrogyria, left hemisphere atrophy and left hippocampal sclerosis. During EEG-fMRI there were frequent left anterior temporal spikes which were linked to a small cluster of left temporal activation (Fig. 2.3). The matching IC corresponded to the GLM activation plus most of the cortical lesion.

Case 4

Patient with left hippocampal sclerosis and left anterior temporal spikes. EEG-fMRI revealed lateral temporal lobe activation. No IC was found that satisfied the matching criteria. 
Table 2

ICA-GLM comparison

\begin{tabular}{lllll}
\hline Case \# & $\begin{array}{l}\text { \# BOLD } \\
\text { IC }\end{array}$ & $\begin{array}{l}\text { \# GLM-concordant } \\
\text { BOLD IC }\end{array}$ & $\begin{array}{l}\text { IC-SPM overlap\% } \\
\text { (\# voxels) }\end{array}$ & $\begin{array}{l}\text { Significance } \\
\text { of } R\end{array}$ \\
\hline 1 & 11 & 1 & $41.5(35)$ & $2.39 \mathrm{e}-06$ \\
2 & 9 & 1 & $18.5(67)$ & 0.009 \\
3 & 24 & 1 & $60(9)$ & 0.01 \\
4 & 21 & 0 & - & - \\
5 & 20 & 2 & $41(500)$ & $3.4 \mathrm{e}-09$ \\
& & & $23(280)$ & $1.7 \mathrm{e}-07$ \\
6 & 13 & 1 & $78(207)$ & $2.0 \mathrm{e}-20$ \\
7 & 17 & 1 & $25(671)$ & $2.6 \mathrm{e}-15$ \\
8 & 11 & 1 & $15(2)$ & $2.6 \mathrm{e}-04$ \\
\hline
\end{tabular}

The volume of overlap between IC maps and SPMs is expressed both as a percentage of the SPM activation cluster. The significance of the correlation between IC time course and spike regressor is expressed as the probability of getting the same value of correlation coefficient by chance.

\section{Case 5}

Patient with two large heterotopic nodules, frontoparietocentral and medial parietal. During EEG-fMRI there were frequent right central spikes or varying amplitudes plus slow waves. GLM activation clusters were observed within both nodules. Two IC matching the concordance criteria were found: one in the nodules (Fig. 2.5a) and the other under the vertex (Fig. 2.5b).

\section{Case 6}

Patient had left hemisphere chronic encephalitis of adult onset. The GLM activation was linked to high-amplitude left temporal sharp-wave discharges. A single matching IC was found covering most of the GLM-derived activation (Fig. 2.6).

\section{Case 7}

Patient had a post-traumatic right middle frontal gyrus scar. EEG-fMRI revealed three distinct clusters of activation linked to runs of polyspike-wave activity. A single matching IC was found with excellent correspondence to the GLM result (Fig. 2.7).

\section{Case 8}

Patient had widespread predominantly posterior band and nodular heterotopia and bursts of posterior temporal/occipital discharges with left-sided emphasis. EEG-fMRI revealed a small cluster of left temporal-occipital activation concordant with structural MRI and interictal EEG. Matching IC consisted of a small cluster overlaying the GLM result (Fig. 2.8).

\section{Discussion}

We analyzed the results of ICA decomposition of fMRI data in patients with focal epilepsy in whom EEG-fMRI had revealed GLM-derived activation patterns judged to be concordant with the epileptic focus. Using automated component classification, in $7 / 8$ cases it was possible to identify one or two IC which corresponded to GLM activations.

\section{Methodological issues}

The classifier we used was trained on data from healthy subjects in a block-designed visual experiment, which may be suboptimal for application in patient data where spontaneous brain activity is recorded without external stimulation. Visual inspection of the components classified as BOLD by expert observers revealed that $50 \%$ of these did not correspond to BOLD effects but rather to motion, big blood vessels and high frequency noise. This represents a trade-off in favor of classifier sensitivity over specificity.

The classifier failed to detect a matching BOLD-related component in a single case (\#4). In this case, inspection of all IC by expert observers revealed a component that qualitatively matched the GLM result that had been classified as temporal high frequency noise. This case was characterized by the highest degree of correlation between motion expressed as displacement in $3 \mathrm{D}$ space calculated with realignment parameters and the GLM regressor $(R=-0.3, p<0.01)$. It should be noted that typical patterns for motion and temporal high frequency noise have similar values for the high frequency parameter of the fingerprint (De Martino et al., 2007) which caused misclassification of the IC. Fragments of the time course of the selected IC and IED-based regressor are shown in Fig. 1a $\left(R=0.25, p=6.6 \mathrm{e}^{-12}\right)$.

In patients, fMRI data are often contaminated by motion and so caution is required when analyzing and interpreting results, especially when applying data-driven approaches. For example, in case \# 2 with 108/450 scans modeled as head jerks, there was considerable overlap between a number of motion-related IC and the GLM result. The presence of motion-related noise risks masking genuine signal changes, particularly when temporally correlated with the events of interest. In case \#4, we concluded that the presence of motion artefact (highly significant correlation of IED-based regressor and integral motion parameter $R=-0.3, p=2.6 \mathrm{e}-15)$ caused misclassification of the GLM-concordant IC (Fig. 2.4) due to high temporal frequency contamination.

We evaluated each BOLD IC based on spatial overlap with GLM activation pattern and temporal correlation with the GLM IED-derived regressor (Fig. 1b). Although the choice of spatial overlap and temporal correlation threshold values can appear arbitrary the results provide some reassurance that this approach is useful.

\section{Interpretation of BOLD components}

One of the key elements of our approach was a data reduction strategy to reduce the number of IC to be matched with the results. The first step of this was the automatic classification of IC as BOLD components. The second step was the visual identification of IC with spatial patterns typical of Resting State Networks: visual, auditory, sensory-motor and so-called Default Mode, in line with previous studies of resting state fMRI data using data-driven techniques (Seifritz et al., 2002; Beckmann et al., 2005; Fransson, 2005; Fox et al., 2005; Aragri et al., 2006; Laufs et al., 2006). We note the asymmetry in the DMN in case \#2 which may reflect pathology of the right hemisphere (Fig. 3.2).

The remaining BOLD IC formed the set of components which comprised epilepsy-related components (in 7/8 cases) and components for which we could not find an interpretation. Examples of such IC are shown in Fig. 4. Some of the uninterpreted IC (i.e. that did not satisfy both the spatial and temporal matching criteria) had a time course that was significantly correlated with the IED-derived GLM regressor, providing evidence that those IC could describe some parts of the spatial distribution of epileptogenic network. This is equivalent to sub- 
threshold activity from the point of view of the GLM and may therefore simply reflect differences in the way significance is established for the two methods. As an additional analysis, we studied the temporal relationship between the 'Other' (potentially epileptic) IC and the relevant GLM regressor as a function of relative time lag. This has revealed significant correlation in 5 of the 7 cases in which potentially epileptic components were identified, with peak correlation at time lags ranging between -1 TR and 2 TR. For example, in case \#5 the correlation peaked at a time lag of +1 TR, suggesting delayed BOLD changes contralateral to the presumed focus in relation to the ipsilateral BOLD effect (as revealed by the GLM) (Fig. 5). The significance of such lags, which may in part reflect normal variability in the hemodynamic response which may be captured using a more flexible HRF basis set, remains to be studied. Nonetheless, the additional components identified in this study illustrate an interesting aspect of data-driven analyses: that of possible added value compared to the GLM-based analysis by removing the need for tight temporal coupling between scalp EEG event and hemodynamic fluctuations. Validation of such components would require correlation with the complete characterization of the epileptogenic network as may be obtained with intracranial EEG. Our assumption is that epileptiform activity recorded on the scalp, and therefore which originates in the superficial cortex, does not fundamentally differ from activity that is generated deeper inside the brain (and that is not reflected on scalp EEG) at least hemodynamically. No additional potentially epilepsy-related IC were found for cases 7 and 8 .

As mentioned previously, in case \#4 no candidate IC matched the EEG-derived pattern. Inspection of the components classified as non-BOLD revealed one for which both the spatial and temporal correspondence criteria were satisfied (Fig. 2.4): spatial overlap between the IC map and the SPM was 93\% (96 voxels) of the SPM volume, the correlation of the IC time course and the IED-based regressor was $R=0.26$ with a very high significance ( $p=6.6 \mathrm{e}-12$, Fig. 1a). As suggested above, this IC was probably contaminated by motion.

One of the two concordant IC in case \#5 (Fig. 2.5b) was observed in all datasets; in principle, there are two possible explanations namely that the component is commonly linked to resting state activity or to epilepsy. Its location close to the sagittal sinus, and distribution pattern suggest the former.
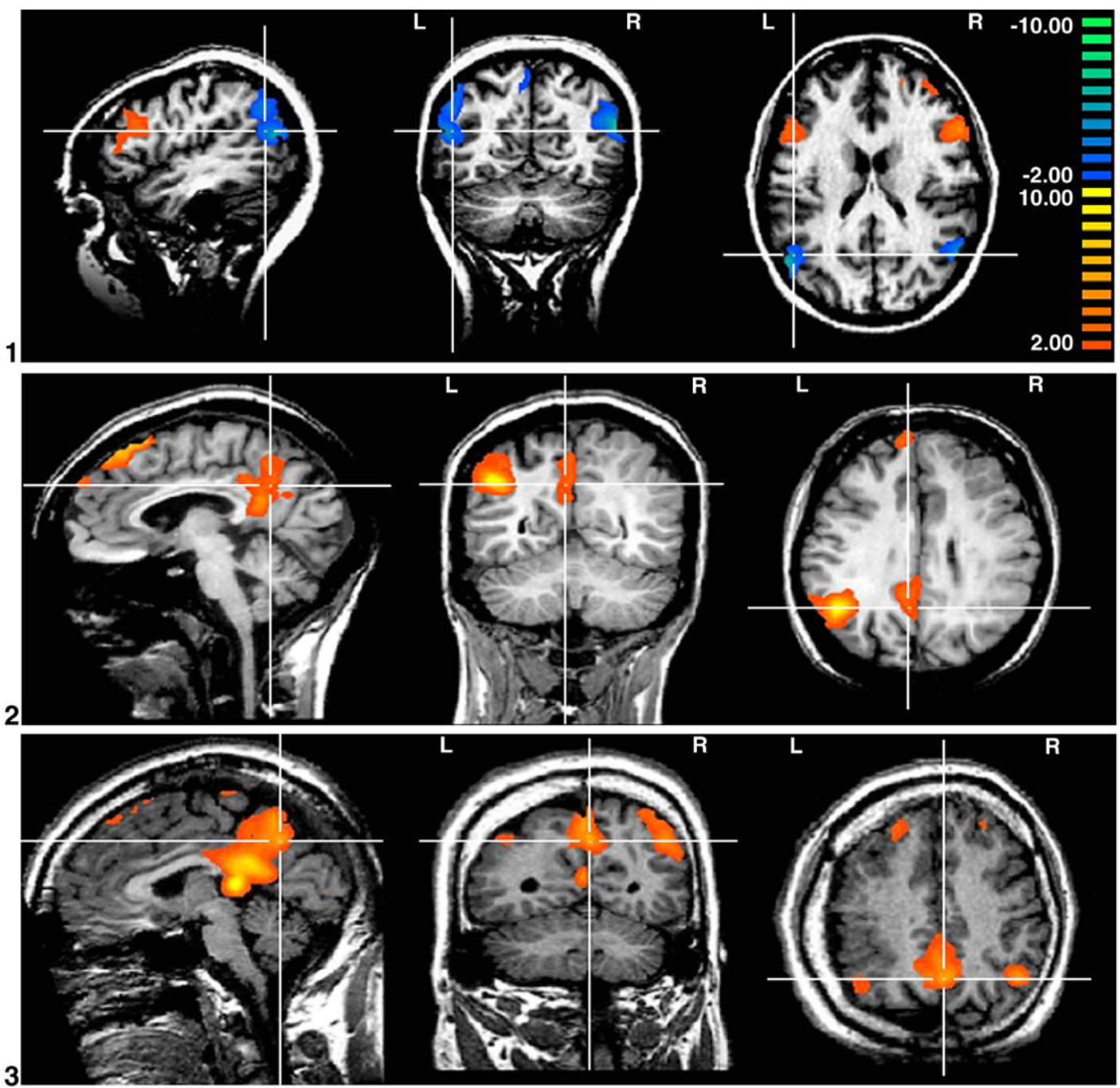

Fig. 3. Illustrative IC maps of components corresponding to resting state networks (Greicius et al., 2003; Fransson, 2005). Each IC map is visualized at a threshold of $z>2.0$; the numbers for each set of projections correspond to \# of case ID in Tables 1 and 2. 

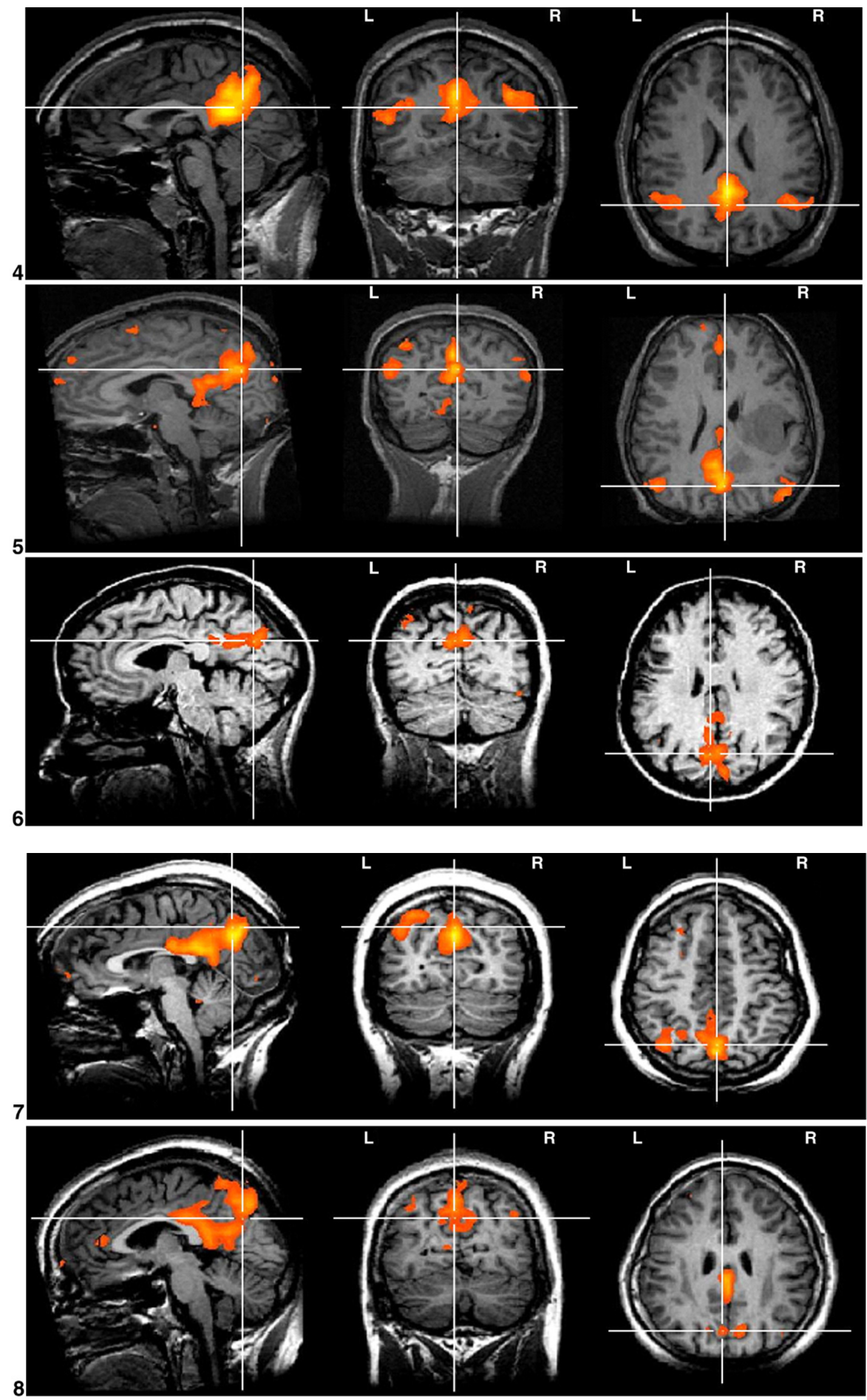


\section{Biological and clinical significance}

The nature of the selected cases allows us to conclude that the matching of ICA and GLM results confirms a general concordance between the identified component(s) and the generator of the IED. Patients 1, 4 and 6 were seizure free 4, 2 and 9 years respectively after left temporal lobe resection. Comparison of the fMRI and post-operative structural MRI revealed that the activated areas were resected, providing strong evidence that ICA was capable of highlighting areas of brain involved in epileptic activity in those cases.

It is important to note that none of the GLM and IC results matched perfectly. For example, only a small degree of spatial overlap between GLM results and IC maps does not preclude functional correspondence. The possible explanations for differences in spatial distribution and time course include: differences in the underlying mathematical assumptions between the models; differences in criteria for selecting significant voxels (thresholding); bias of the GLM analysis due to limited EEG sensitivity, EEG bias towards more superficial activity and subjectivity of the EEG interpretation. Thus in case \#4 when increasing the threshold up to $z>3.0$ and further to $z>4.0$ there was still significant spatial overlap with the SPM, at $85 \%$ and $52 \%$ of SPM volume correspondingly. The spatial configuration of the IC maps and the SPM appeared more similar to each other with these higher thresholds than for the case of $z>2.0$ shown in Fig. 2.4.

\section{Future work}

The ability of ICA to blindly decompose the time series in a set of independent (and physiologically meaningful) components makes it an attractive tool to formulate spatial hypotheses with respect to the source of IED. ICA alone, however, does not allow distinguishing IED-related activity from other BOLD related activation patterns (e.g. default mode and resting state patterns). The next step would be to perform ICA of fMRI in patients in whom no EEG-derived model is available due to the absence of epileptic activity in the EEG data acquired during EEG-fMRI acquisition. In such cases the GLM approach is not applicable and it will be necessary to develop new methods based on non temporal
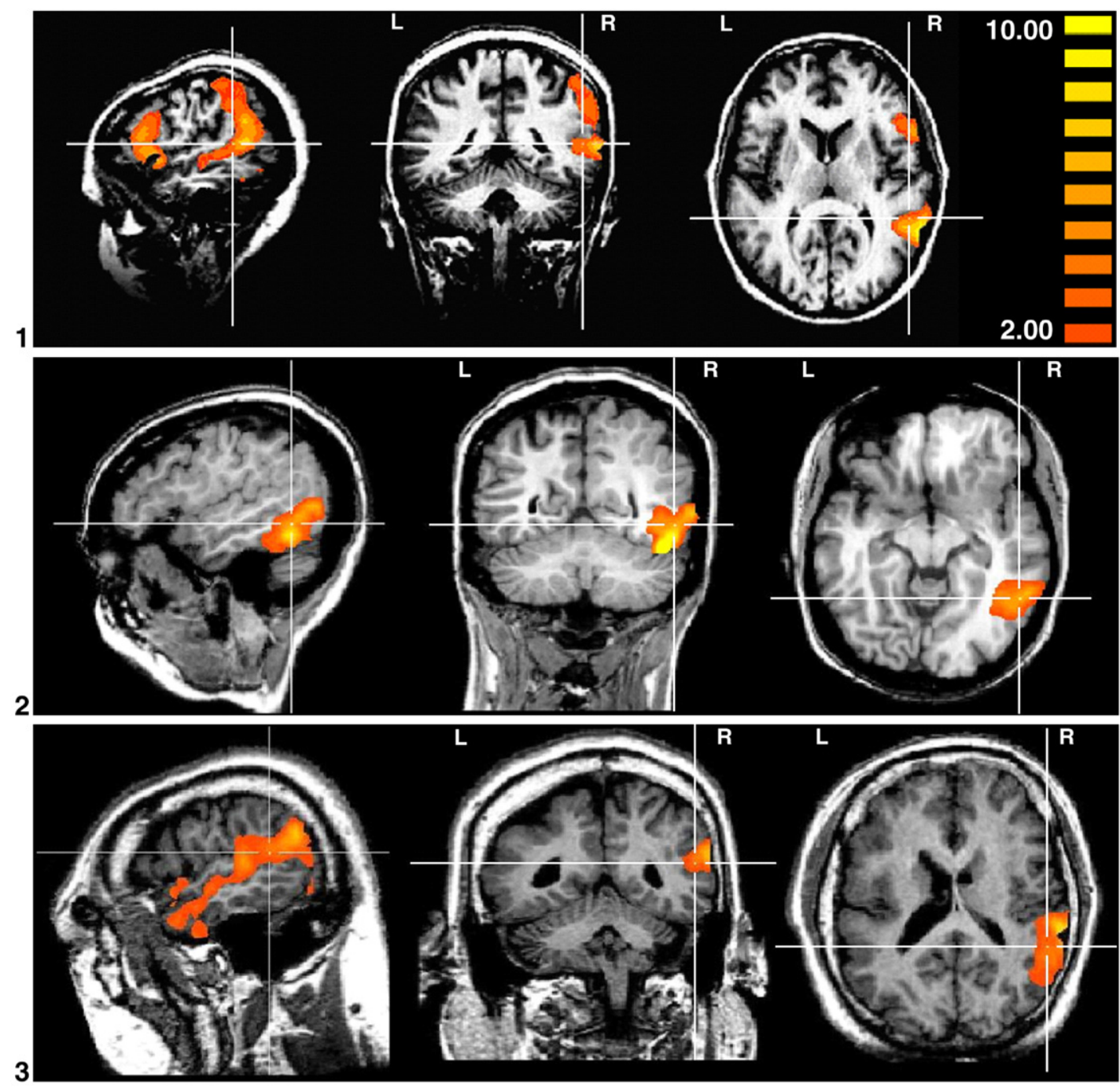

Fig. 4. Representative illustration of IC requiring further investigation and interpretation for cases 1-6. Visualization threshold $z>2.0$. Case 1: contralateral to the GLM result; case 2: posterior to the GLM result; case 3: contralateral to the lesion and GLM result; case 4: posterior to the GLM result; case 5: contralateral to the lesion and GLM result; case 6: contralateral to the lesion and GLM result. The numbers for each set of projections correspond to \# of case in Tables 1 and 2. 

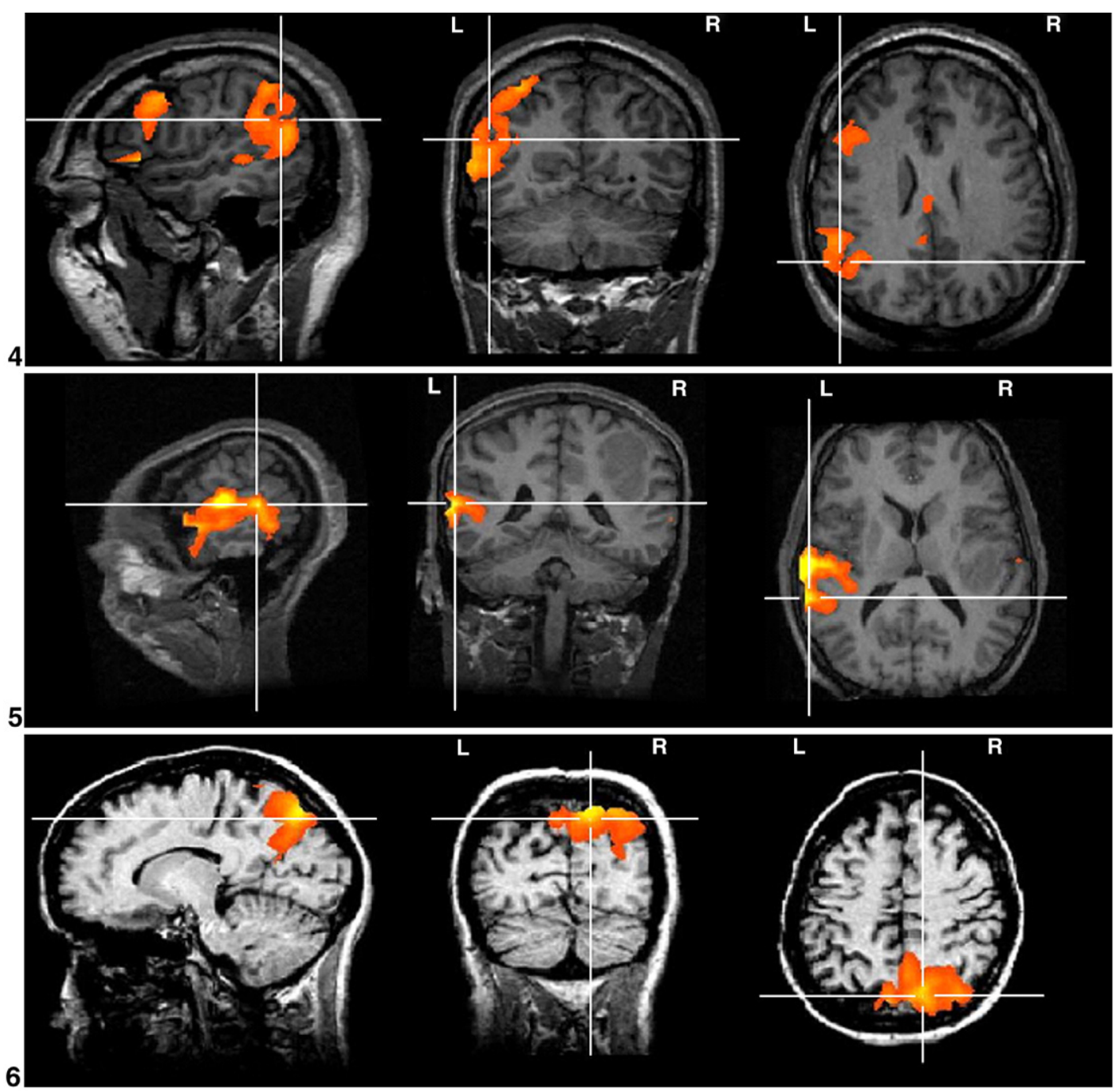

Fig. 4 (continued).

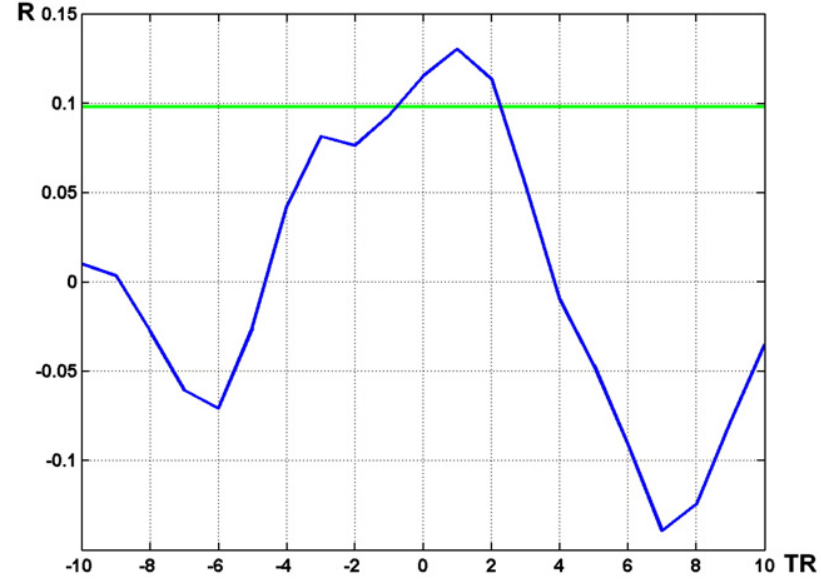

Fig. 5. Illustration of correlation function between IED-derived regressor and time course of an IC interpreted as 'Other' (case \#5). The map of the IC is shown in Fig. 4.5. The level of significance of correlation is $R=0.98$ (green line). Here peak correlation at +1 TR means a delay of the IC time course in relation to IED-derived regressor. priors in order to determine which IC are epilepsy-related. Retraining or refinement of the classifier according to resting state data in healthy subjects or epileptic patients may allow increased specificity. Additional information such as longer duration EEG recordings outside the scanner, intra-cranial EEG and surgical data may also be used. This could lead to a methodology for further reducing the number of candidate epileptiform components. Validation could be performed based on comparison with information derived from intra-cranial EEG data and post-surgical outcome.

\section{Conclusions}

We found that ICA of interictal fMRI is capable of revealing areas of epileptic activity in patients with well-characterized focal epilepsy. The automatic IC classification method used in this study resulted in a limited number of IC, hypothesized to correspond to epileptic activity. In all cases studied at least one of the IC was consistent with EEG-based BOLD activations and due to the nature of the patients selected represent epileptic regions 
that generate IED. In a number of cases, IC were revealed that did not correspond to the EEG-derived result but that could be the basis for further investigation, and in particular by comparison with results of other diagnostic modalities, to assess the method's ability to identify pathological changes not linked to scalp EEG abnormalities.

\section{Acknowledgments}

This work was funded by the Medical Research Council (grant number G0301067; RR and DC), the Wellcome Trust (grant number 067176; LL), the Bundesministerium für Bildung und Forschung (HL) and Deutsche Forschungsgemeinschaft grant LA 1452/3-1 (HL). Thanks to Mark Symms for MRI developments, Philippa Bartlett and Jane Burdett for MRI scanning. The support of the National Society for Epilepsy (UK) and Brain Innovation (Netherlands) is acknowledged.

\section{References}

Allen, P.J., Polizzi, G., Krakow, K., Fish, D.R., Lemieux, L., 1998. Identification of EEG Events in the MR Scanner: the Problem of Pulse Artefact and a Method for Its Subtraction. Neuroimage 8, 229-239.

Allen, P.J., Josephs, O., Turner, R., 2000. A method for removing imaging artifact from continuous EEG recorded during functional MRI. NeuroImage 12, 230-239.

Aragri, A., Scarabino, T., Seifritz, E., Comani, S., Cirillo, S., Tedeschi, G., Esposito, F., Di Salle, F., 2006. How does spatial extent of fMRI datasets affect independent component analysis decomposition? Hum. Brain Mapp. 27, 736-746.

Beckmann, C.F., Smith, S.M., 2004. Probabilistic independent component analysis for functional magnetic resonance imaging. IEEE Trans. Med. Imag. 23 (2), 137-152.

Beckmann, C.F., DeLuca, M., Devlin, J.T., Smith, S.M., 2005. Investigations into resting-state connectivity using independent component analysis. Philos. Trans. R. Soc. Lond., B Biol. Sci. 360 (1457), 1001-1013.

Benar, C.G., Gross, D.W., Wang, Y.H., Petre, V., Pike, B., Dubeau, F., Gotman, J., 2002. The BOLD response to interictal epileptiform discharges. NeuroImage 17, 1182-1192.

Chen, H., Yao, D., Lu, G., Zhang, Z., Hu, Q., 2006. Localization of latent epileptic activities using spatio-temporal independent component analysis of fMRI data. Brain Topogr. 19 (1-2), 21-28.

De Martino, F., Gentile, F., Esposito, F., Balsi, M., Di Salle, F., Goebel, R., Formisano, E., 2007. Classification of fMRI independent components using IC-fingerprints and support vector machine classifiers. NeuroImage 34 (1), 177-194.

Di Bonaventura, C., Vaudano, A., Carnì, M., Pantano, P., Nucciarelli, V., Garreffa, G., Maraviglia, B., Prencipe, M., Bozzao, L., Manfredi, M., Giallonardo, A., 2006. EEG/fMRI study of ictal and interictal epileptic activity: methodological issues and future perspectives in clinical practice. Epilepsia 47 (s5), 52-58.

Diehl, B., Salek-Haddadi, A., Fish, D.R., Lemieux, L., 2003. Mapping of spikes, slow waves, and motor tasks in a patient with malformation of cortical development using simultaneous EEG and fMRI. Magn. Reson. Imaging 21 (10), 1167-1173.

Frackowiak, R.S.J., Friston, K.J., Frith, C., Dolan, R., Price, C.J., Zeki, S., Ashburner, J., Penny, W.D., 2003. Human Brain Function, 2nd edition. Academic Press.

Fransson, P., 2005. Spontaneous low-frequency BOLD signal fluctuations: an fMRI investigation of the resting-state default mode of brain function hypothesis. Hum. Brain Mapp. 26 (1), 15-29.
Friston, K.J., Williams, S., Howard, R., Frackowiak, R.S., Turner, R., 1996. Movement-related effects in fMRI time-series. Magn. Reson. Med. 35 (3), 346-355.

Formisano, E., Esposito, F., Kriegeskorte, N., Tedeschi, G., Di Salle, F., Goebel, R., 2002. Spatial independent component analysis of functional magnetic resonance imaging time-series: characterization of the cortical components. Neurocomputing 49, 241-254.

Formisano, E., Esposito, F., Di Salle, F., Goebel, R., 2004. Cortex-based independent component analysis of fMRI time-series. Magn. Reson. Imaging 22 (10), 1493-1504.

Fox, M.D., Snyder, A.Z., Vincent, J.L., Corbetta, M., Van Essen, D.C., Raichle, M.E., 2005. The human brain is intrinsically organized into dynamic, anticorrelated functional networks. Proc. Natl. Acad. Sci. U. S. A. 102 (27), 9673-9678.

Greicius, M.D., Menon, V., 2004. Default-mode activity during a passive sensory task: uncoupled from deactivation but impacting activation. J, Cogn. Neurosci. 16 (9), 1484-1492 (Nov).

Greicius, M.D., Krasnow, B., Reiss, A.L., Menon, V., 2003. Functional connectivity in the resting brain: a network analysis of the default mode hypothesis. Proc. Natl. Acad. Sci. U. S. A. 100 (1), 253-258.

Gotman, J., Benar, C.G., Dubeau, F., 2004. Combining EEG and FMRI in epilepsy: methodological challenges and clinical results. J. Clin. Neurophysiol. 21 (4), 229-240.

Gotman, J., Kobayashi, E., Bagshaw, A.P., Benar, C.G., Dubeau, F., 2006. Combining EEG and fMRI: a multimodal tool for epilepsy research. J. Magn. Reson. Imaging 23 (6), 906-920.

Hamandi, K., Salek-Haddadi, A., Fish, D.R., Lemieux, L., 2004. EEG/ functional MRI in epilepsy: the Queen Square experience. J. Clin. Neurophysiol. 21 (4), 241-248.

Hamandi, K., Salek Haddadi, A., Liston, A., Laufs, H., Fish, D.R., Lemieux, L., 2005. fMRI temporal clustering analysis in patients with frequent interictal epileptiform discharges: comparison with EEGdriven analysis. NeuroImage 26 (1), 309-316.

Hawco, C.S., Bagshaw, A.P., Lu, Y., Dubeau, F., Gotman, J., 2007. BOLD changes occur prior to epileptic spikes seen on scalp EEG. NeuroImage 35 (4), 1450-1458.

Hyvärinen, A., 1999. Fast and robust fixed-point algorithms for independent component analysis. IEEE Trans. Neural Netw. 10 (3), 626-634.

Hu, D., Yan, L., Liu, Y., Zhou, Z., Friston, K., Tan, C., Wu, D., 2005. Unified SPM-ICA for fMRI analysis. NeuroImage 25, 746-755.

Krakow, K., Woermann, F.G., Symms, M.R., Allen, P.J., Lemieux, L., Barker, G.J., Duncan, J.S., Fish, D.R., 1999. EEG-triggered functional MRI of interictal epileptiform activity in patients with partial seizures. Brain 122, 1679-1688.

Laufs, H., Duncan, J.S., 2007. Electroencephalography/functional MRI in human epilepsy: what it currently can and cannot do. Curr. Opin. Neurol. 20 (4), 417-423.

Laufs, H., Holt, J.L., Elfont, R., Krams, M., Paul, J.S., Krakow, K., Kleinschmidt, A., 2006. Where the BOLD signal goes when alpha EEG leaves. NeuroImage 31, 1408-1418.

Lemieux, L., Salek-Haddadi, A., Josephs, O., Allen, P., Toms, N., Scott, C., Krakow, K., Turner, R., Fish, D.R., 2001. Event-related fMRI with simultaneous and continuous EEG: description of the method and initial case report. NeuroImage 14, 780-787.

Lemieux, L., Salek-Haddadi, A., Lund, T.E., Laufs, H., Carmichael, D., 2007. Modelling large motion events in fMRI studies of patients with epilepsy. Magn. Reson. Imaging 25 (6), 894-901.

McKeown, M.J., 2000. Detection of consistently task-related activations in fMRI data with hybrid independent component analysis. NeuroImage $11,24-35$.

McKeown, M.J., Makeig, S., Brown, G.G., Jung, T.P., Kindermann, S.S., Bell, A.J., Sejnowski, T.J., 1998. Analysis of fMRI data by blind separation into independent spatial components. Hum. Brain Mapp. 6, 160-188.

McKeown, M.J., Hansen, L.K., Sejnowski, T.J., 2003. Independent component analysis of functional MRI: what is signal and what is noise? Curr. Opin. Neurobiol. 13, 620-629. 
Mirsattari, S.M., Wang, Z., Ives, J.R., Bihari, F., Leung, L.S., Bartha, R., Menon, R.S., 2006. Linear aspects of transformation from interictal epileptic discharges to BOLD fMRI signals in an animal model of occipital epilepsy. NeuroImage 30, 1133-1148.

Morgan, V.L., Price, R.R., Arain, A., Modur, P., Abou-Khalil, B., 2004. Resting functional MRI with temporal clustering analysis for localization of epileptic activity without EEG. NeuroImage 21, 473-481.

Niedermeyer, E., Lopes da Silva, F., 2004. Electroencephalography: Basic Principles, Clinical Applications, and Related Fields, 1st edition. Williams \& Wilkins.

Ray, A., Tao, J.X., Hawes-Ebersole, S.M., Ebersole, J.S., 2007. Localizing value of scalp EEG spikes: a simultaneous scalp and intracranial study. Clin. Neurophysiol. 118 (1), 69-79.

Salek-Haddadi, A., Friston, K.J., Lemieux, L., Fish, D.R., 2003. Studying spontaneous EEG activity with fMRI. Brain Res. Rev. 43, 110-133.

Salek-Haddadi, A., Diehl, B., Hamandi, K., Merschhemke, M., Liston, A., Friston, K., Duncan, J.S., Fish, D.R., Lemieux, L., 2006. Hemodynamic correlates of epileptiform discharges: an EEG-fMRI study of 63 patients with focal epilepsy. Brain. Res. 1088 (1), 148-166.

Schmithorst, V.J., Brown, R.D., 2004. Empirical validation of the triple-code model of numerical processing for complex math operations using functional MRI and group independent component analysis of the mental addition and subtraction of fractions. NeuroImage 22, 1414-1420.

Seifritz, E., Esposito, F., Hennel, F., Mustovic, H., Neuhoff, J.G., Bilecen, D., Tedeschi, G., Scheffler, K., Di Salle, F., 2002. Spatiotemporal pattern of neuronal processing in the human auditory cortex. Science 297, 1706-1708.

Tao, J.X., Ray, A., Hawes-Ebersole, S., Ebersole, J.S., 2005. Intracranial EEG substrates of scalp EEG interictal spikes. Epilepsia 46 (5), 669-676.

Van de Ven, V.G., Formisano, E., Prvulovic, D., Roeder, C.H., Linden, D. E., 2004. Functional connectivity as revealed by spatial independent component analysis of fMRI measurements during rest. Hum. Brain Mapp. 22 (3), 165-178. 